\title{
Modelling of Shear Banding in Plane Couette Flow of Non-linear Viscoelastic Fluids
}

\author{
David Kibe Muchiri ${ }^{1}$, Isaac Chepkwony ${ }^{2}$ \\ ${ }^{1}$ African Institute For Mathematical Sciences, University of the Western Cape, \\ South Africa. Email: davidkibe@aims.ac.za \\ ${ }^{2}$ Department of Mathematics \& Actuarial Science, Kenyatta University, Kenya
}

\begin{abstract}
We investigate shear banding phenomena in the plane Couette flow of viscoelastic fluids.The viscoelastic fluids are modelled via the Giesekus constitutive equations with stress diffusion. The nonlinear and coupled systems of equations, comprising the momentum equation and the constitutive equations, are solved numerically using semi-implicit finite difference methods. The effects of the fluid parameters on the flow variables are also investigated. Under certain values of the material parameters, we observe formation of shear bands in plane Couette flow.
\end{abstract}

Keywords: Couette flow, Giesekus model, Shear band,Viscoelastic fluid.

\section{INTRODUCTION}

In the recent years, it has been observed that the flow of viscoelastic fluids display unusual flow behavior even in simple geometries. Some of these unusual flow patterns result from complex flow instabilities such as shear banding. Shear banding is characterized by an apparent discontinuity in the flow profile which occur when shear rate takes two distinct values corresponding to low and high shear rate. Discontinuity in the shear rate arise when a fluid under shear forces is deformed beyond a certain critical point [1]. Shear banding has been observed to occur in shear flow of viscoelastic fluids such as wormlike micelles, foams, polymeric gels, polymer melts, emulsions and granular materials. These materials have recently drawn interest to researchers due to their wide range of applications and great relevance in science, medicine, engineering and technology $[2,3]$. Even though shear banding has always been physically observed in real flows of viscoelastic fluids, there has been an incomplete understanding of their origins and formations. This paper, thus, explores shear banding phenomena with the aim of enhancing the understanding of their rheological behavior.

There exists a review work on various model types used to study viscoelasticity and are well summarized by [4, 5]. In this paper, however, we choose to use the Giesekus model discussed by Joseph [6], which has been widely used in the rheology of viscoelastic fluids. The Giesekus model has attracted a lot of interest in the past because of its three main advantages. Firstly, the Giesekus model presents constitutive law which provides a mechanism to control the elongational property of viscoelastic materials. Secondly, the Giesekus model provides realistic behavior for both shear flows and shear-free flows. It predicts well the shear thinning, shear viscosity and other behavior of complex flows. Lastly, the model is explicitly described by two material parameters; the zero-shear- rate viscosity $\eta_{s}$ and the stress relaxation time $\lambda$, together with dimensionless anisotropymobility factor $\alpha$, [7]. However, the model is characterized by a quadratic term in the stress tensor which makes it non-linear.

The rheology of viscoelastic fluids has been widely studied, for instance, Baumert and Muller [8] gave a considerable attention to elastic Taylor-Couette instability in Boger fluids in a bid to understand viscoelastic instabilities. Shear flow instabilities and their origin have been discussed and summarized by Wilson et al. [9]. Polymer processing of materials such as polymer melts, plastics and other synthetic fiber products together with low Reynolds number instabilities involved have been reviewed by Larson [10]. Wormlike micelles have been widely used to investigate complex instabilities in viscoelastic fluids, for instance, Team [11] conducted a research on shear banding of aqueous wormlike micellar solution and gave a summary of their complex micro-structural properties of viscosity and elasticity. Britton et al. [12] used conventional rheometry and velocimetry to 


\section{International Journal of Advances in Scientific Research and Engineering (ijasre), Vol 6 (2), February-2020}

investigate shear banding in Couette flow of viscoelastic materials and observed dynamical complex instabilities in shear bands. There is a vast literature on numerical methods for solving complex equations of viscoelastic flows, [13, 14]. For instance, the theory of constitutive equations for unsteady flows of viscoelastic materials and their numerical solutions using finite difference schemes were summarized by Tom et al. [15]. Formulation of finite difference methods for structured grids involving higher order terms have been documented by Hirsch [16]. Despite the intensive research and numerous efforts, the numerical approximations of viscoelastic flows still poses a challenge to researchers due to the nonlinearity and flow instabilities caused by the elasticity and viscosity behavior of the fluid. The objective of this review is to use the Giesekus model to obtain numerical approximations of viscoelastic flows and analyze shear banding instability in planar Couette flow, by using finite difference method for both space and time.

\section{THE GIESEKUS MODEL}

Giesekus introduced the constitutive law (2.3) expressed in terms of the upper convected derivative [6], which together with the continuity equation (2.1) and momentum equation (2.2) completes the model. We thus use the following system of equations of the Giesekus model to describe the motion of an incompressible viscoelastic fluid in terms of the conformation tensor;

- $\quad$ Continuity equation:

$$
\nabla \cdot \overline{\mathrm{v}}=0
$$

- $\quad$ Cauchy equation:

$$
\rho \frac{\partial \overline{\mathrm{v}}}{\partial t}=-\rho \overline{\mathrm{v}} \cdot \nabla \overline{\mathrm{v}}-\nabla p+\nabla \cdot \overline{\bar{\sigma}}
$$

- $\quad$ Time evaluation equation for the conformation tensor:

$$
\frac{\partial \overline{\bar{c}}}{\partial t}=-\overline{\mathrm{v}} \cdot \nabla \overline{\overline{\mathrm{c}}}+\overline{\overline{\mathrm{c}}} \cdot \nabla \overline{\mathrm{v}}+(\nabla v)^{T} \cdot \overline{\overline{\mathrm{c}}}-\frac{1}{\lambda}\left[(1-\alpha) \overline{\overline{\mathrm{I}}}+\alpha \frac{\mathrm{K}}{\mathrm{K}_{\mathrm{B}} \mathrm{T}} \overline{\overline{\mathrm{c}}}\right] \cdot\left[\overline{\overline{\mathrm{c}}}-\frac{\mathrm{K}}{\mathrm{K}_{\mathrm{B}} \mathrm{T}} \overline{\overline{\mathrm{I}}}\right]+D \nabla^{2} \overline{\overline{\mathrm{c}}}
$$

where $\overline{\mathrm{v}}$ is the velocity of the fluid, $\rho$ is the total mass density, $p$ the pressure, $\overline{\bar{\sigma}}$ the deviatoric stress, $\overline{\overline{\mathrm{c}}}$ the conformation tensor, $\lambda$ the characteristic relaxation time, $\alpha$ the anisotropy factor for hydrodynamic interactions, $D$ diffuson coefficient, $\eta_{\mathrm{s}}$ solvent viscosity, $\mathrm{K}$ the spring constant, $\mathrm{K}_{\mathrm{B}}$ and $\mathrm{T}$ are the viscoelastic parameters. The fourth term on the right hand side of equation (2.3) represents the nonlinear relaxation term while the fifth term corresponds to the stress diffusive term.

Deviatoric stress is given by;

$$
\overline{\bar{\sigma}}=\overline{\bar{\tau}}+2 \eta_{\mathrm{s}} D^{\prime}
$$

where $\overline{\bar{\tau}}=G_{0}(\xi \overline{\overline{\mathrm{c}}}-\overline{\overline{\mathrm{I}}})$ is the polymer stress tensor, $G_{0}$ the modulus of elasticity, $\xi=\frac{\mathrm{K}}{\mathrm{K}_{\mathrm{B}} \mathrm{T}}$ and $D^{\prime}=\frac{1}{2}\left[\nabla \overline{\mathrm{V}}+(\nabla v)^{T}\right]$ is the deformation rate of strain. The initial and boundary conditions prescribed at the solid walls are discussed in the next section.

We use the Giesekus model in the subsequent section to analyze the shear banding formed in a viscoelastic fluid flowing in a Couette flow configuration in which the fluid moves between two moving parallel plates. The two wall boundaries can be moving in the same or opposite direction with uniform velocity $\overline{\mathrm{u}}_{1}$ and $\overline{\mathrm{u}}_{2}$ respectively.

\section{PLANE COUETTE FLOW}

We consider a Couette flow in a 2D-rectangular coordinate system directed in the $x$ direction as shown below, with the upper plate moving with a velocity $\bar{U}$ and the lower plate fixed. 


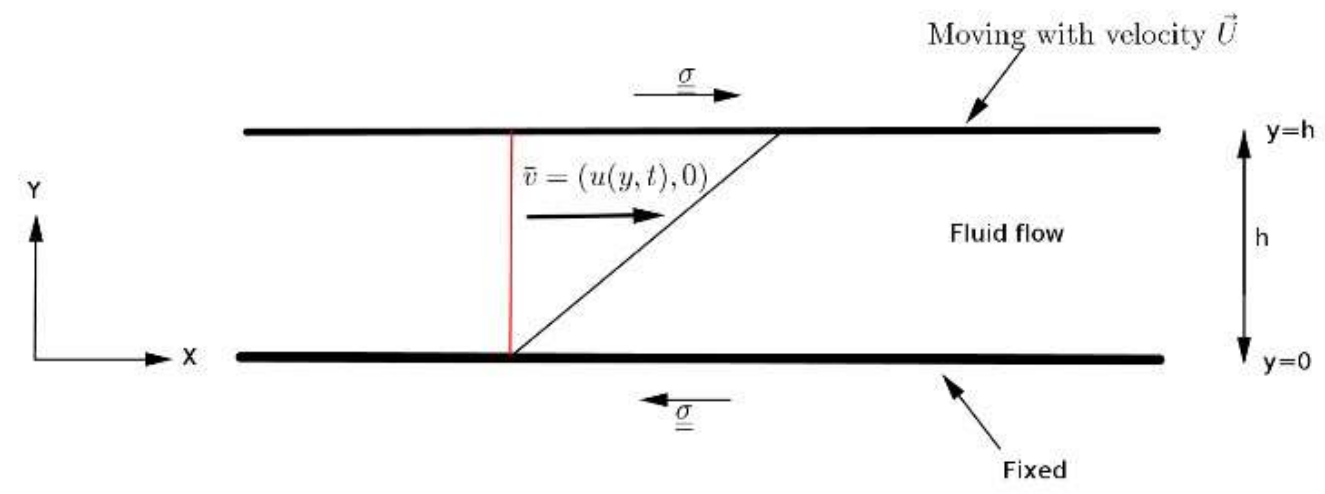

Figure 1: Plane Couette flow

Since the flow is in Cartesian system with $x$ in the stream-wise direction and $y$ in the transverse direction, we take $\overline{\mathrm{v}}=(u(y), 0), \quad \frac{\partial p}{\partial y}=0 \quad$ and $\quad \frac{\partial()}{\partial x}=0$

We now write the governing equations in Cartesian form.

Continuity equation (2.1) becomes;

$$
\frac{\partial u}{\partial y}=0
$$

An explicit expression for deviatoric stress is given by

$$
\overline{\bar{\sigma}}=G_{0}(\xi \overline{\bar{c}}-\overline{\bar{I}})+\eta_{s}\left[\nabla \overline{\mathrm{v}}+(\nabla v)^{T}\right]
$$

In Cartesian form this expression reduces into a system of three equations by symmetry:

$$
\begin{gathered}
\sigma_{x x}=G_{0}\left(\xi c_{x x}-1\right) \\
\sigma_{x y}=G_{0} \xi c_{x y}+\eta_{\mathrm{s}} \frac{\partial u}{\partial y} \\
\sigma_{y y}=G_{0}\left(\xi c_{y y}-1\right)
\end{gathered}
$$

Using the above equations, the conservation of momentum (2.2) in Cartesian form becomes;

$$
\rho \frac{\partial u}{\partial t}=G_{0} \xi \frac{\partial c_{x y}}{\partial y}+\eta_{\mathrm{s}} \frac{\partial^{2} u}{\partial y^{2}}
$$

The time evolution equation for the conformation tensor given by (2.3) can be rewritten as;

$$
\frac{\partial \overline{\bar{c}}}{\partial t}+\overline{\mathrm{v}} \cdot \nabla \overline{\overline{\mathrm{c}}}-\overline{\overline{\mathrm{c}}} \cdot \nabla-(\nabla v)^{T} \cdot \overline{\overline{\mathrm{c}}}=-\frac{1}{\lambda}\left[(1-\alpha) \overline{\overline{\mathrm{I}}}+\alpha \frac{\mathrm{K}}{\mathrm{K}_{\mathrm{B}} \mathrm{T}} \overline{\overline{\mathrm{c}}}\right] \cdot\left[\overline{\overline{\mathrm{c}}}-\frac{\mathrm{K}}{\mathrm{K}_{\mathrm{B}} \mathrm{T}} \overline{\overline{\mathrm{I}}}\right]+D \nabla^{2} \overline{\overline{\mathrm{c}}}
$$

The terms on the left hand side of the equal sign represents the upper-convected time derivative. On the right hand side we have, the linear term, constant, non-linear term and diffusion term respectively. Expanding these terms individually, Equation (3.3) gives us a matrix equation which by symmetry reduces into a system of three time evolution equations for the conformation tensor: 


$$
\begin{aligned}
& \frac{\partial c_{x x}}{\partial t}=-\frac{\alpha \xi}{\lambda}\left(c_{x x}^{2}+c_{x y}^{2}\right)-\frac{1}{\lambda}\left(1-\alpha-\alpha \xi^{2}\right) c_{x x}+\frac{1}{\lambda}(1-\alpha) \xi+D \frac{\partial^{2} c_{x x}}{\partial y^{2}} \\
& \frac{\partial c_{x y}}{\partial t}=c_{x x} \frac{\partial u}{\partial y}-\frac{\alpha \xi}{\lambda}\left(c_{x x} c_{x y}+c_{x y} c_{y y}\right)-\frac{1}{\lambda}\left(1-\alpha-\alpha \xi^{2}\right) c_{x y}+D \frac{\partial^{2} c_{x y}}{\partial y^{2}} \\
& \frac{\partial c_{y y}}{\partial t}=2 c_{y x} \frac{\partial u}{\partial y}-\frac{\alpha \xi}{\lambda}\left(c_{x y}^{2}+c_{y y}^{2}\right)-\frac{1}{\lambda}\left(1-\alpha-\alpha \xi^{2}\right) c_{y y}+\frac{1}{\lambda}(1-\alpha) \xi+D \frac{\partial^{2} c_{y y}}{\partial y^{2}}
\end{aligned}
$$

Considering one wall is fixed we have the initial conditions: $\bar{u}(\mathrm{y}, 0)=0$ and $\overline{\bar{\tau}} \rightarrow 0$ as $t \rightarrow 0$, the no slip conditions: $\bar{u}(0, \mathrm{t})=0$ and $\bar{u}(\mathrm{~h}, \mathrm{t})=\overline{\mathrm{U}}$, together with the stress condition: $\frac{\partial \tau}{\partial y}=0$ at the walls.

\section{METHOD OF SOLUTION}

We approximate the solution of the governing equations using finite difference method. We first define a set of grid points in our domain $D$ such that $(y, t) \in D$. Assuming a uniformly spaced interval $0 \leq y \leq h$ and a uniform time interval $0 \leq t \leq T$, where $h$ is the length and $T$ the maximum time, we make the following choices;

- State step size $\Delta y=\frac{h-0}{N}$

- Time step size $\Delta t=\frac{T}{M}$

We denote the solution in the point $\left(y_{j}, t_{n}\right)$ by $u_{j}^{n}=u\left(y_{j}, t_{n}\right)$ and assume that $\Delta y=\Delta t$ and $N+1$ is the total number of spatial points. When discretizing the governing equations, we assume that the solution at the current time step $t_{n}$ is known and $t_{n+1}$ is unknown. We thus collect similar terms and bring all the unknowns to the left and the known to the right.

To obtain the numerical scheme for Cauchy equation we discretize equation (3.2) as follows:

$$
\frac{u_{j}^{n+1}-u_{j}^{n}}{\Delta t}=\frac{G_{0} \xi}{\rho}\left[\frac{\left[c_{x y}\right]_{j+1}^{n}-\left[c_{x y}\right]_{j-1}^{n}}{2 \Delta y}\right]+\frac{\eta_{s}}{2 \rho}\left[\frac{u_{j+1}^{n+1}-2 u_{j}^{n+1}+u_{j-1}^{n+1}}{(\Delta y)^{2}}+\frac{u_{j+1}^{n}-2 u_{j}^{n}+u_{j-1}^{n}}{(\Delta y)^{2}}\right]
$$

This gives us;

$$
u_{j}^{n+1}-u_{j}^{n}=\frac{G_{0} \xi \Delta t}{2 \rho \Delta t}\left[\left[c_{x y}\right]_{j+1}^{n}-\left[c_{x y}\right]_{j-1}^{n}\right]+\frac{\eta_{s} \Delta t}{2 \rho(\Delta y)^{2}}\left[u_{j+1}^{n+1}-2 u_{j}^{n+1}+u_{j-1}^{n+1}+u_{j+1}^{n}-2 u_{j}^{n}+u_{j-1}^{n}\right]
$$

We let $\quad k_{1}=\frac{G_{0} \xi \Delta t}{2 \rho \Delta t}$ and $k_{2}=\frac{\eta_{\mathrm{s}} \Delta t}{2 \rho(\Delta y)^{2}}$

Collecting all terms involving $t_{n+1}$ time step to the left we obtain the required scheme:

$$
\begin{array}{r}
-k_{2} u_{j+1}^{n+1}+\left(1+2 k_{2}\right) u_{j}^{n+1}-k_{2} u_{j-1}^{n+1}= \\
k_{1}\left[\left[c_{x y}\right]_{j+1}^{n}-\left[c_{x y}\right]_{j-1}^{n}\right]+k_{2} u_{j+1}^{n}+\left(1-2 k_{2}\right) u_{j}^{n}+k_{2} u_{j-1}^{n}
\end{array}
$$

In a similar way, numerical schemes for time evolution equation (3.4), (3.5) and (3.6) respectively are obtained below.

$$
\begin{gathered}
-R_{1}\left[c_{x x}\right]_{j-1}^{n+1}+\left(1+2 R_{1}\right)\left[c_{x x}\right]_{j}^{n+1}-R_{1}\left[c_{x x}\right]_{j+1}^{n+1}=\left[c_{x x}\right]_{j}^{n}-\frac{\Delta t \alpha \xi}{\lambda}\left(\left(\left[c_{x x}\right]_{j}^{n}\right)^{2}+\left(\left[c_{x y}\right]_{j}^{n}\right)^{2}\right)-\frac{\Delta t}{\lambda}\left[1-\alpha-\alpha \xi^{2}\right]\left[c_{x x}\right]_{j}^{n}+ \\
\frac{\Delta t}{\lambda}(1-\alpha) \xi+R_{1}\left(\left[c_{x x}\right]_{j+1}^{n}-2\left[c_{x x}\right]_{j}^{n}+\left[c_{x x}\right]_{j-1}^{n}\right)
\end{gathered}
$$




$$
\begin{gathered}
-R_{1}\left[c_{x y}\right]_{j-1}^{n+1}+\left(1+2 R_{1}\right)\left[c_{x y}\right]_{j}^{n+1}-R_{1}\left[c_{x y}\right]_{j+1}^{n+1}=\frac{\Delta t}{2 \Delta y}\left[c_{x x}\right]_{j}^{n}\left[u_{j+1}^{n}-u_{j-1}^{n}\right]-\frac{\Delta t \alpha \xi}{\lambda}\left(\left[c_{x x}\right]_{j}^{n}\left[c_{x y}\right]_{j}^{n}+\left[c_{x y}\right]_{j}^{n}\left[c_{y y}\right]_{j}^{n}\right)+ \\
\left(1-\frac{\Delta t}{\lambda}\left[1-\alpha-\alpha \xi^{2}\right]\right)\left[c_{x y}\right]_{j}^{n}+R_{1}\left(\left[c_{x y}\right]_{j+1}^{n}-2\left[c_{x y}\right]_{j}^{n}+\left[c_{x y}\right]_{j-1}^{n}\right)(4.3) \\
-k_{3}\left[c_{y y}\right]_{j+1}^{n+1}+\left(1+2 k_{3}\right)\left[c_{y y}\right]_{j}^{n+1}-k_{3}\left[c_{y y}\right]_{j-1}^{n+1}=\frac{\Delta t}{\Delta y}\left[c_{y x}\right]_{j}^{n}\left[u_{j+1}^{n}-u_{j-1}^{n}\right]-\frac{\Delta t \alpha \xi}{\lambda}\left(\left(\left[c_{x y}\right]_{j}^{n}\right)^{2}+\left(\left[c_{y y}\right]_{j}^{n}\right)^{2}\right)+\frac{\Delta t}{\lambda}(1-\alpha) \xi+ \\
k_{3}\left[c_{y y}\right]_{j+1}^{n}+\left(1-\frac{\Delta t}{\lambda}\left[1-\alpha-\alpha \xi^{2}\right]-2 k_{3}\right)\left[c_{y y}\right]_{j}^{n}+k_{3}\left[c_{y y}\right]_{j-1}^{n}
\end{gathered}
$$

Where $R_{1}=\frac{D \Delta t}{2(\Delta y)^{2}}$ and $k_{3}=\frac{D \Delta t}{2(\Delta y)^{2}}$

Discretizing the initial and boundary conditions we get the following initial conditions; $u_{j}^{0}=0,\left[c_{x x}\right]_{j}^{0}=\frac{1}{\xi},\left[c_{y y}\right]_{j}^{0}=\frac{1}{\xi}$,

$\left[c_{x y}\right]_{j}^{0}=0$ and boundary conditions: $u_{0}^{n+1}=0$ and $u_{N}^{n+1}=\bar{U}$ where $N=\frac{h}{\Delta y}$, together with the discretized stress conditions at the walls: $\left[c_{x x}\right]_{1}^{n+1}=\left[c_{x x}\right]_{0}^{n+1}$ and $\left[c_{x x}\right]_{N}^{n+1}=\left[c_{x x}\right]_{N-1}^{n+1},\left[c_{x y}\right]_{1}^{n+1}=\left[c_{x y}\right]_{0}^{n+1}$ and $\left[c_{x y}\right]_{N}^{n+1}=\left[c_{x y}\right]_{N-1}^{n+1},\left[c_{y y}\right]_{1}^{n+1}=$ $\left[c_{y y}\right]_{0}^{n+1}$ and $\left[c_{y y}\right]_{N}^{n+1}=\left[c_{y y}\right]_{N-1}^{n+1}$.

\section{EXPERIMENTS AND FITTIN PROCEDURE}

The experimental data we report in this paper is for the non-linear viscoelastic fluids that exhibits shear banding and that are described by the Giesekus model. The values assigned to the parameters of the model were determined as follows: the effective value of time relaxation parameter was determined by fitting the model to small amplitude oscillatory shear data. The effective value of solvent viscosity was determined by adjusting different values until a shear banded velocity profile was obtained while keeping a good agreement with shear stress. The equilibrium breakage point was determined by setting the model to steady state while keeping a good agreement with viscosity and relaxation time. Anisotropy factor was estimated by fitting the model to a steady state while keeping a good agreement with shear stress. A moderate diffusion coefficient was obtained by choosing the value that gave a smooth transition between the shear bands. The values assigned to the parameters used in this model are provided in the table below. We used $N=100$ number of points and time step size $\Delta t=0.01$ all through.

\begin{tabular}{|l|c|c|}
\hline Parameter & Symbol & Value \\
\hline Solvent viscosity & $\eta_{s}$ & $0.099 \mathrm{Pas}$ \\
\hline Modulus of elasticity & $G_{0}$ & $150 \mathrm{~Pa}$ \\
\hline Relaxation time & $\lambda$ & $0.013 \mathrm{~s}$ \\
\hline Anisotropy factor & $\alpha$ & 0.91 \\
\hline Diffusion coefficient & $D$ & $5 \mathrm{~m}^{2} / \mathrm{s}$ \\
\hline Viscoelastic parameter & $\xi$ & 1 \\
\hline
\end{tabular}

Table 1: Values assigned to parameters used in the model.

\section{RESULTS AND DISCUSSION}

In this section we analyze and discuss the results predicted by the Giesekus model for transient and steady shear. Figure 2 shows the variation of the fluid velocity and the stress components with $y$ for the purely viscous Newtonian fluid obtained by setting conformation tensor equal to zero in the full model given by equations (2.1) - (2.3). This result is very important for code validation. By setting conformation tensors equal to zero, all the viscoelastic variables are vanished as expected; hence we recover the predicted linear fluid velocity profile, which is a solution to Equation (3.2). 

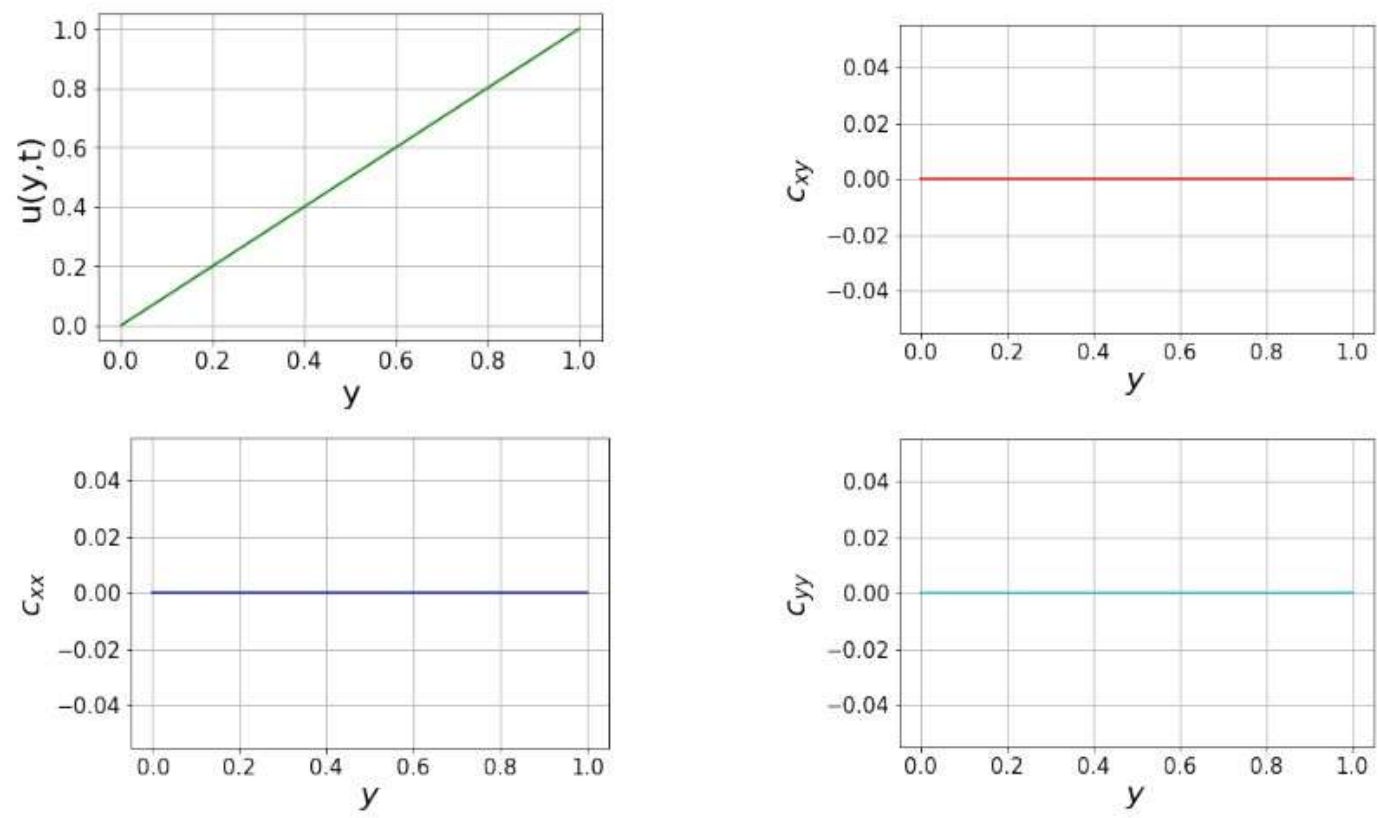

Figure 2: Purely viscous Newtonian fluid obtained by setting conformation tensors equal to zero in our full model. The velocity profile is a straight line and the stress components are all equal to zero.

Figure 3 shows antisymmetric case for a viscoelastic non-Newtonian fluid obtained by setting the lower wall fixed and the upper wall moving with a velocity $U=1 \mathrm{~ms}^{-1}$. Figure 3 a shows fluctuation of the velocity profile which results into two banding regions. The lower band within the range $0.0 m-0.7 m$ of $y$ values, represents low shear rate of a linear and homogeneous viscoelastic flow that occur before deformation. On the other hand, the upper band between $0.9 \mathrm{~m}-1.0 \mathrm{~m}$ represents high shear rate region occurring after deformation. The smooth edge separating the two bands at $y=0.8 \mathrm{~m}$ corresponds to the stress plateau that connects the two bands to ensure continuity.

Figure $3 \mathrm{~b}$ shows a S-shaped shear stress profile within a very small sheared region of almost constant shear stress of about $0.07 \mathrm{~Pa}$. The sheared thickness is a small gap scaled from $0.06 \mathrm{~Pa}-0.08 \mathrm{~Pa}$. The curve between $y=0.0 \mathrm{~m}$ and $y=0.03 m$ corresponds to the transient flow that occur within a short period after a preshear. Moreover, the line between $0.3 \mathrm{~m}$ and $0.9 \mathrm{~m}$ corresponds to the steady state shear that occur around a critical stress of about $C_{x y}=0.07 P a$. When shear rate exceeds characteristic relaxation time required to attain steady state after a disturbance, the profile bocomes unstable as seen between $0.09 \mathrm{~m}$ and $0.10 \mathrm{~m}$.

Figure $3 \mathrm{c}$ shows the first normal stress difference against $y$. Similar to $C_{x y}, C_{y y}$ lies within a too small stress interval of about $1.0045 \mathrm{~Pa}$ to $1.008 \mathrm{~Pa}$, which gives a critical normal stress value of about $C_{y y}=1 P a$. This implies that there is no significant difference in the sheared stress between the two sheared regions.

Clearly, Figure $3 \mathrm{~d}$ shows a constant stress $C_{y y}=1 P a$ which similarly shows that we can have two shear bands with almost same shear stress. We can thus conclude that velocity varies in a wide range of values as shear stress remains around critical shear stress of about $C_{x y}=0.07 \mathrm{~Pa}$ and normal stress of about $C_{x x}=1 P a$ and $C_{y y}=1 P a$. It is thus evidently observed that shear banding does not depend on the separation $y$, hence we can deduce that it is a material property. 


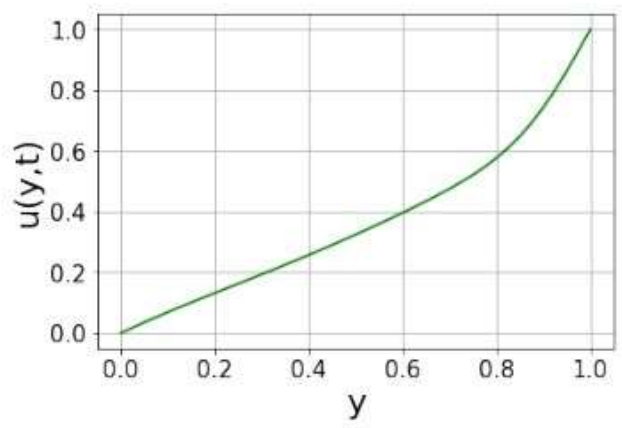

(a)

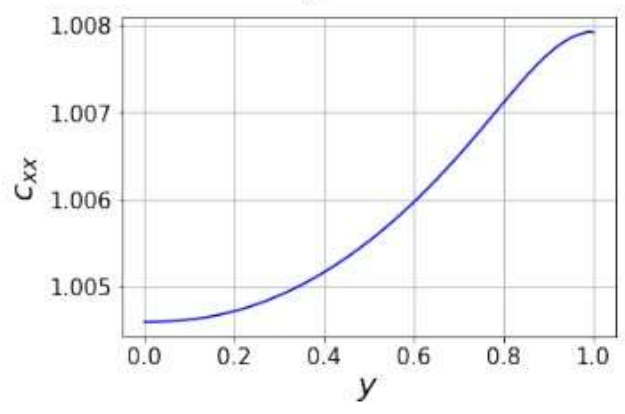

(c)

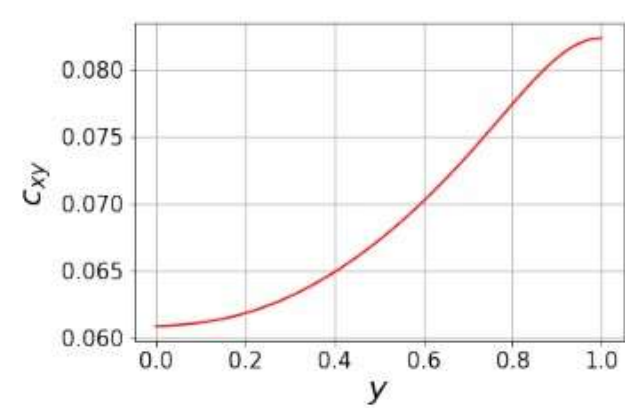

(b)

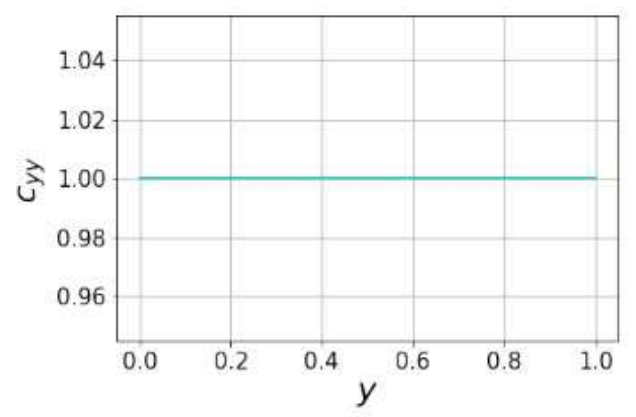

(d)

Figure 3: Antisymmetric case for a viscoelastic fluid obtained by setting the lower wall fixed and the upper wall moving with velocity $U=1 \mathrm{~ms}^{-1}$. Two shear bands are formed in the velocity profile. Shear stress profile is within a very small sheared region of almost constant shear stress. Normal stress profile also lies within a very small gap of almost uniform stress. $C_{y y}$ stays at one Pascal.

Figure 4 shows a symmetric case for the velocity profile and the components of conformation tensor obtained by setting the upper and the lower wall moving in opposite directions with velocities $U=1 \mathrm{~ms}^{-1}$ and $U=-1 \mathrm{~ms}^{-1}$ respectively. The velocity profile, in Figure 4a shows coexistence of three sheared regions flowing at different shear rates but almost similar shear stress. The lower band from $0.0 m-0.2 m$ and the topmost band from $0.8 m-1.0 m$ have the same velocity and corresponds to high shear rate. The middle band on the other hand is a linear region corresponding to low shear rate. To ensure continuity, the three bands are separated by a smooth stress plateau. Figures $4 \mathrm{~b}$ and $4 \mathrm{c}$ show catenary-shaped curves of shear stress $c_{x y}$ and normal stress $c_{x x}$ respectively. In both cases the time evolution of shear stress is depicted clearly from the transient state to steady state region. The left hand side of the curve shows adecreasing shear stress connected smoothly with an increasing shear stress on the right. The decreasing shear stress corresponds to $U=-1 \mathrm{~ms}^{-1}$ while the increasing shear stress corresponds to $U=1 \mathrm{~ms}^{-1}$. But all the same, the sheared region is within a very small interval as shown in $c_{x y}$ profile. Figure $4 \mathrm{~d}$ gives a straight horizontal line at $c_{y y}=1$ which corresponds to $\xi=1$. This is a similar result to antisymmetric case discussed above. As a matter of fact, the diagonal component of conformation $c_{y y}$ stays at a constant equal to 1 for all our computation. 


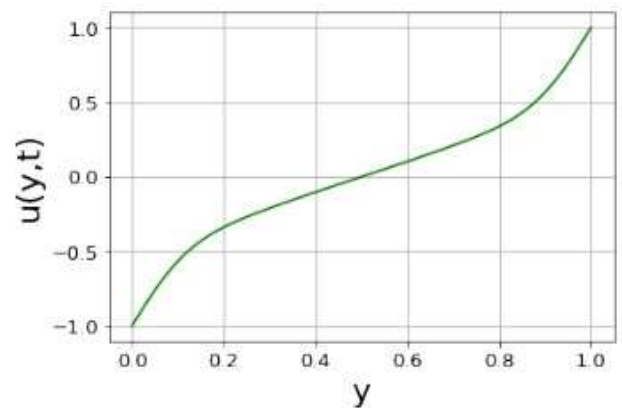

(a)

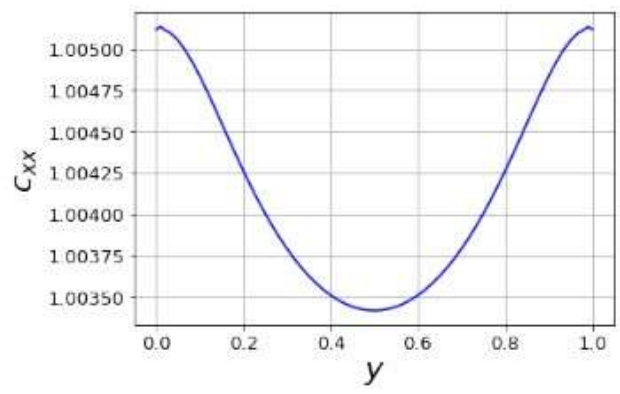

(c)

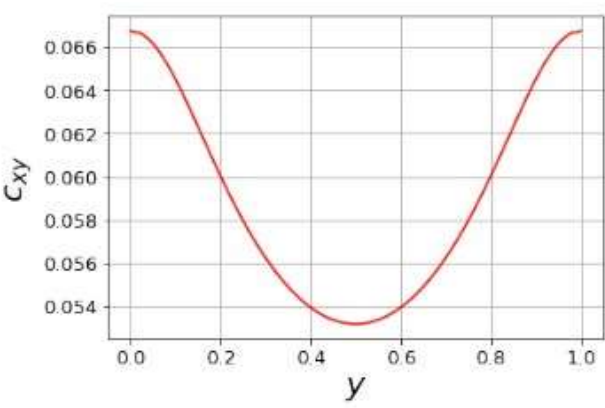

(b)

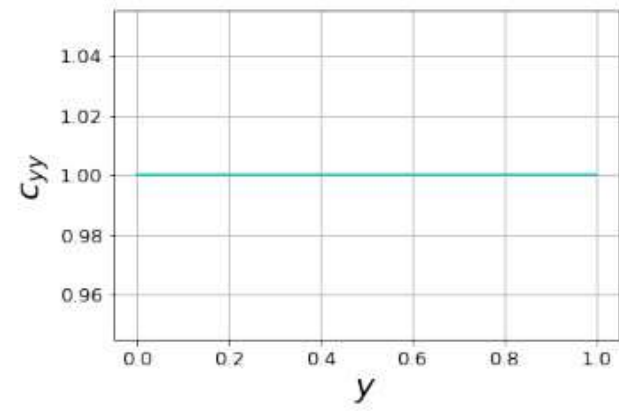

(d)

Figure 4: Symmetric case for a viscoelastic fluid obtained by setting the upper and the lower wall moving with velocity $U=1 \mathrm{~ms}^{-1}$ and $U=-1 \mathrm{~ms}^{-1}$ respectively. Three shear bands are formed in the velocity profile. Both shear and normal stress profiles lie within a very small gap of almost constant shear stress.

Figure 5 and 6 show the development of steady solution with time for the entire flow profile. We notice convergence of the maximum flow quantities for both antisymmetric and symmetric cases. For antisymmetric case all the solutions attain steady state at time $t<100$ while for symmetric case all the solutions attain steady state at time $t<50$. For the velocity profile in Figure 5, the curve at $t=1 \mathrm{~s}$ appear to have a very sharp bend but with time the bend reduces until a steady solution is attained at $t=100 \mathrm{~s}$, which corresponds to 10000 time steps using $\Delta t=0.01$. For velocity profile in Figure 6 , the curve at $t=1 \mathrm{~s}$ appear to also have a very sharp bend but with time the bend reduces until a steady solution is attained at $t=50 \mathrm{~s}$, which corresponds to 5000 time steps using $\Delta t=0.01$. The velocity profile increases smoothly until a steady state is attained. For the components of conformation tensor, the shear profiles at initial time $t=1 \mathrm{~s}$ appear to oscillate with a large amplitude compared to the amplitude at steady state. For the diagonal component $c_{y y}$, the profile remains constant. The model predicts the overshoot of the shear stress occurring during the initial stage of the non-linear deformation. The development of the steady solution with time and the convergence of the entire flow profile imply that these solutions are independent of time. 

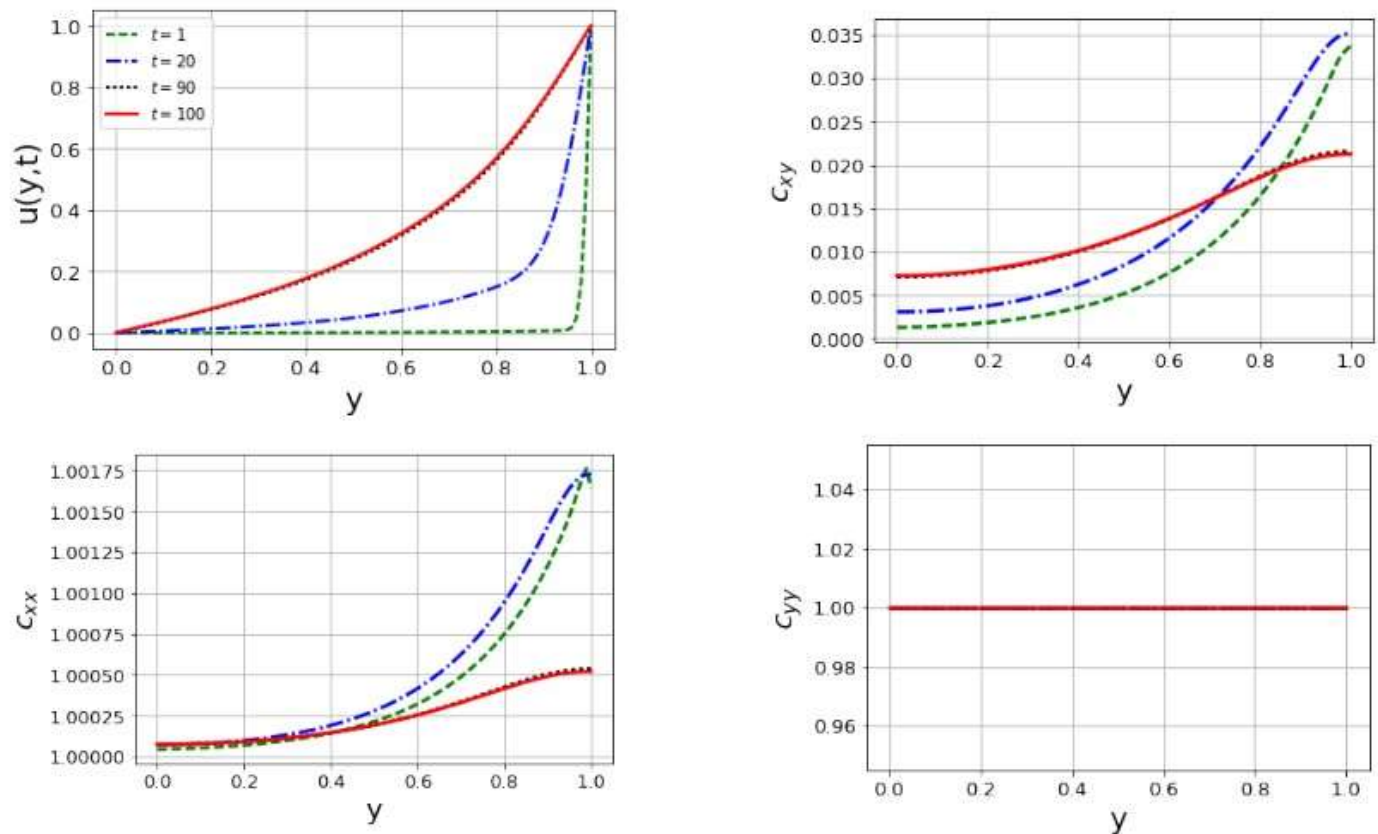

Figure 5: Development of steady flow profiles with $\Delta t=0.01$ in the antisymmetric case. Entire flow profiles are converged at $t<100 s$.
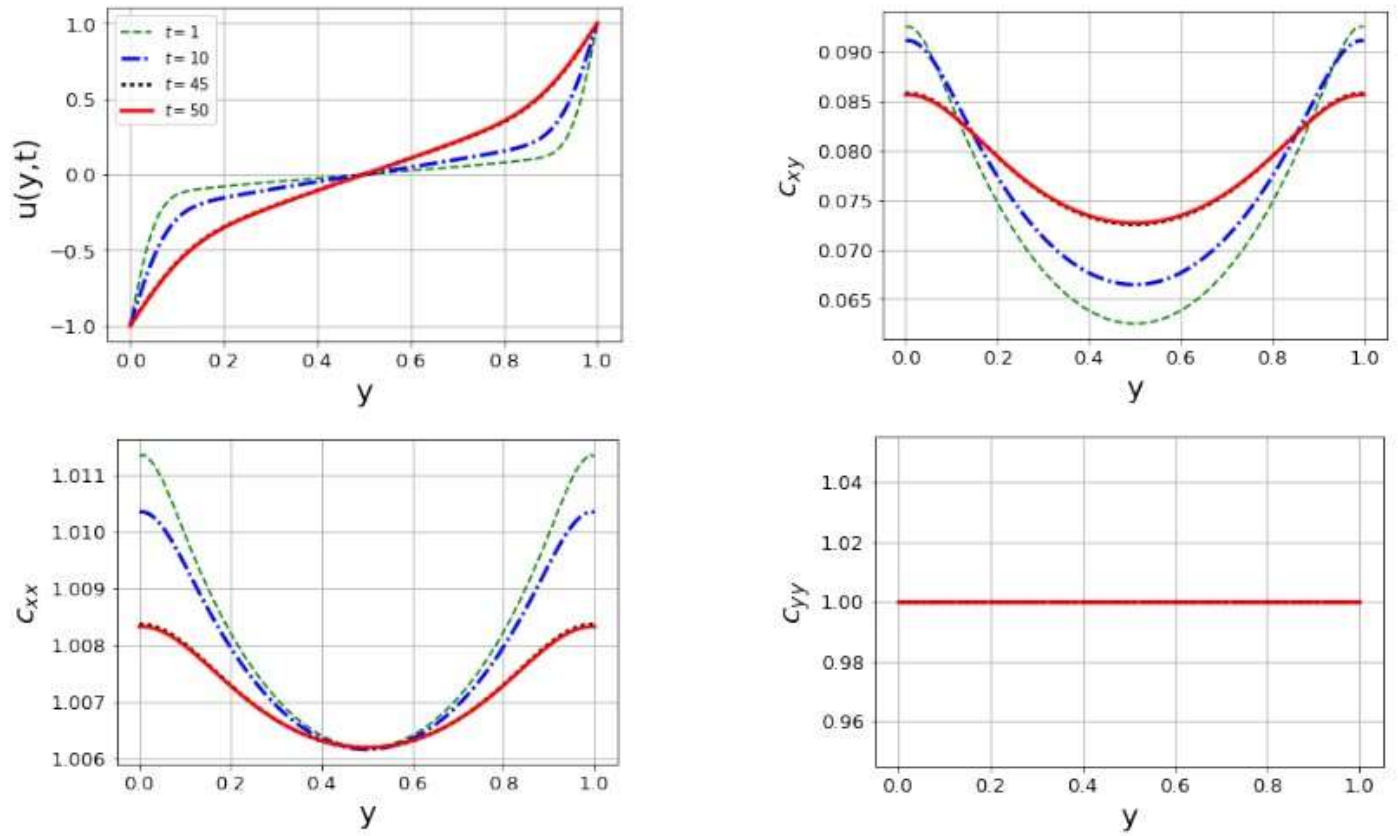

Figure 6: Development of steady flow profiles in the symmetric case with $\Delta t=0.01$. Entire flow profiles converge at $t<50 s$.

So far we have used a fixed set of values for the flow parameters. We now give a broad picture of the dependence of solutions on flow parameters. We only consider antisymmetric configuration for all parameter dependence solutions. Figures $7-14$ respectively, show the dependence of maximum fluid velocities and conformation tensor components with the diffusivity parameter $D$, concentration $\rho$, anisotropy factor $\alpha$, relaxation time parameter $\lambda$, elasticity parameter $G_{0}$, a viscoelastic parameter $\xi$ and velocity $\bar{U}$. All graphs are plotted using parametric values provided in Table 1 . 
Figure 7 shows the variation of flow quantities with the diffusion coefficient. As expected, the parameter $D$ in Equation (2.3) increases the stress components together with the viscous term which in turn reduces the velocity. As seen in the velocity profile, it's clear that the magnitude of diffusion coefficient determines the transition between the two bands. A large diffusivity cause a large bend in the velocity curve whereas lowering diffusivity makes the curve smooth. In all these cases $c_{y y}$ stays at a constant equal to $1 \mathrm{~Pa}$ all through.

Figure 8 shows that high level of concentration $\rho$, reduces the velocity but increases the intensity of the stress components. This was clearly expected from Equation (3.3) where density lowers velocity by increasing viscosity and stress. As a matter of fact, density is the dominating parameter with a wide range of values.

As Figure 9 displays, the parameter $\alpha$ shows no significant effects on the fluid velocity and on shear stress $c_{x y}$. Since $\alpha$ is not directly attached to the velocity equation nor the viscous terms in the constitutive equation, its only observable effect is on the normal stress component $c_{x x}$. It affects the intensity of the normal stress.

A slight increase in the parameter $\lambda$ increases the velocity and the intensity of stress components as shown in Figure 10. In absence of the relaxation effect, shear banding does not occur. As expected from Equation (3.2), $G_{0}$ increases the velocity but reduces the intensity of stress components. This is well depicted in Figure 11. As we expected, the parameter $\eta_{s}$, leads to an increase in the fluid velocity and a decrease in the viscous term of Equation (3.2). This in turn decreases the stress intensity as observed in Figure 12. Parameter $\xi$ lowers both fluid velocity and shear stress $c_{x y}$ as seen in Figure 13, but increases normal stresses $c_{x x}$ and $c_{y y}$. Both $c_{x x}$ and $c_{y y}$ attain different constant values corresponding to $\xi$.

As seen in Figure 14, high velocity corresponds to an increase in the shear rate which in turn increases the intensity of the stress components. The resultant effect of high shear rate is an increase in the rate of deformation which causes shear banding.
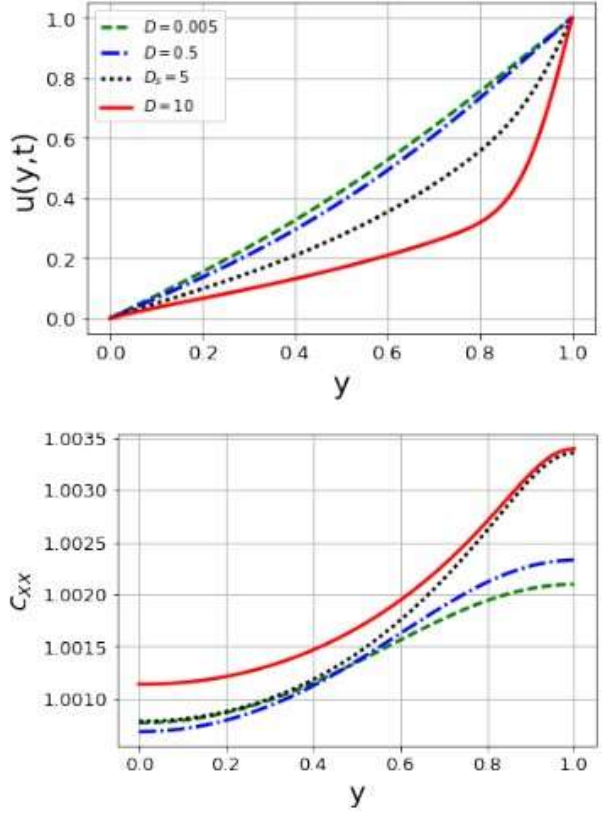
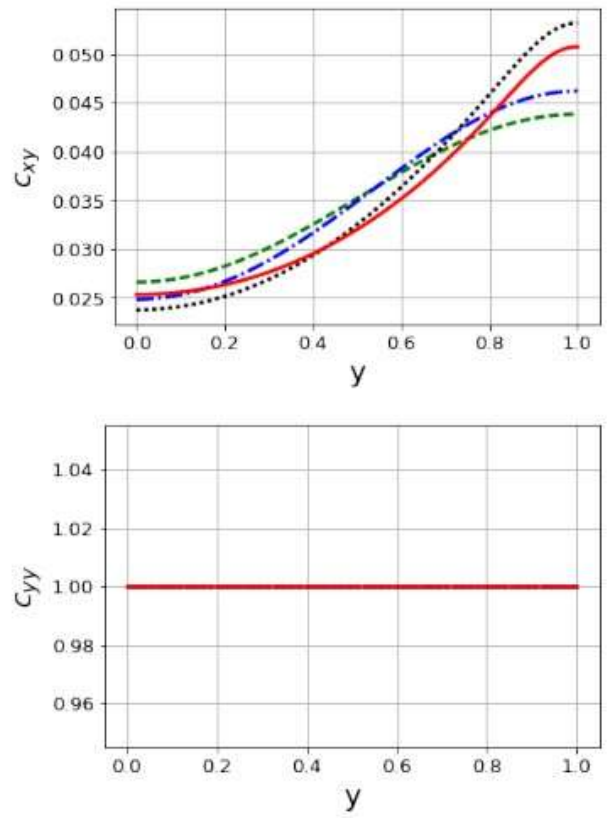

Figure 7: Variation of flow quantities with the diffusion coefficient $D$. $D$ smoothens the transition region between the two shear bands and increases the intensity of stress components. 

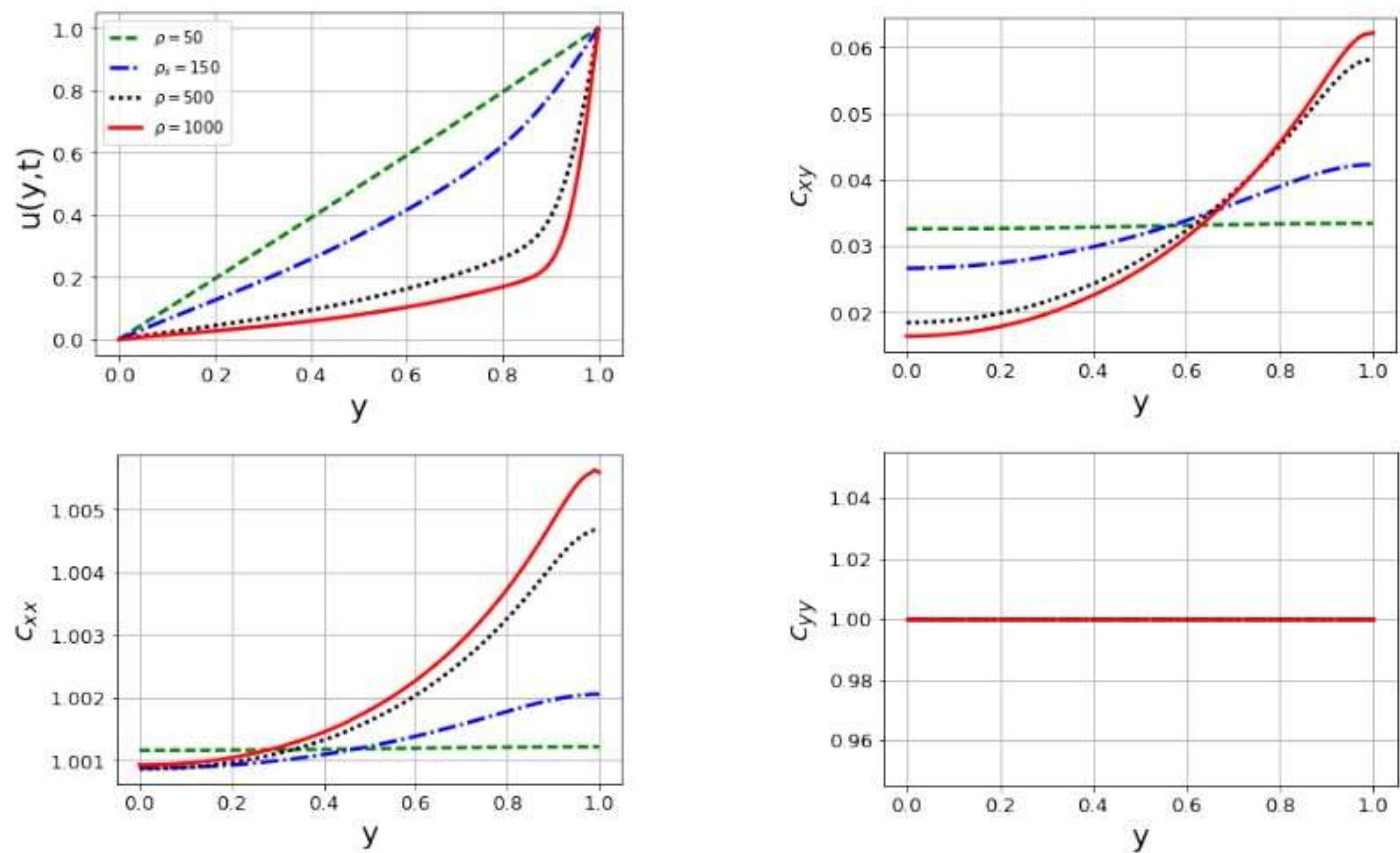

Figure 8: Variation of flow quantities with fluid concentration $\rho$. Density $\rho$, lowers the fluid velocity but increases the intensity of stress components $c_{x y}$ and $c_{x x}$. $c_{y y}$ stays at one Pascal.
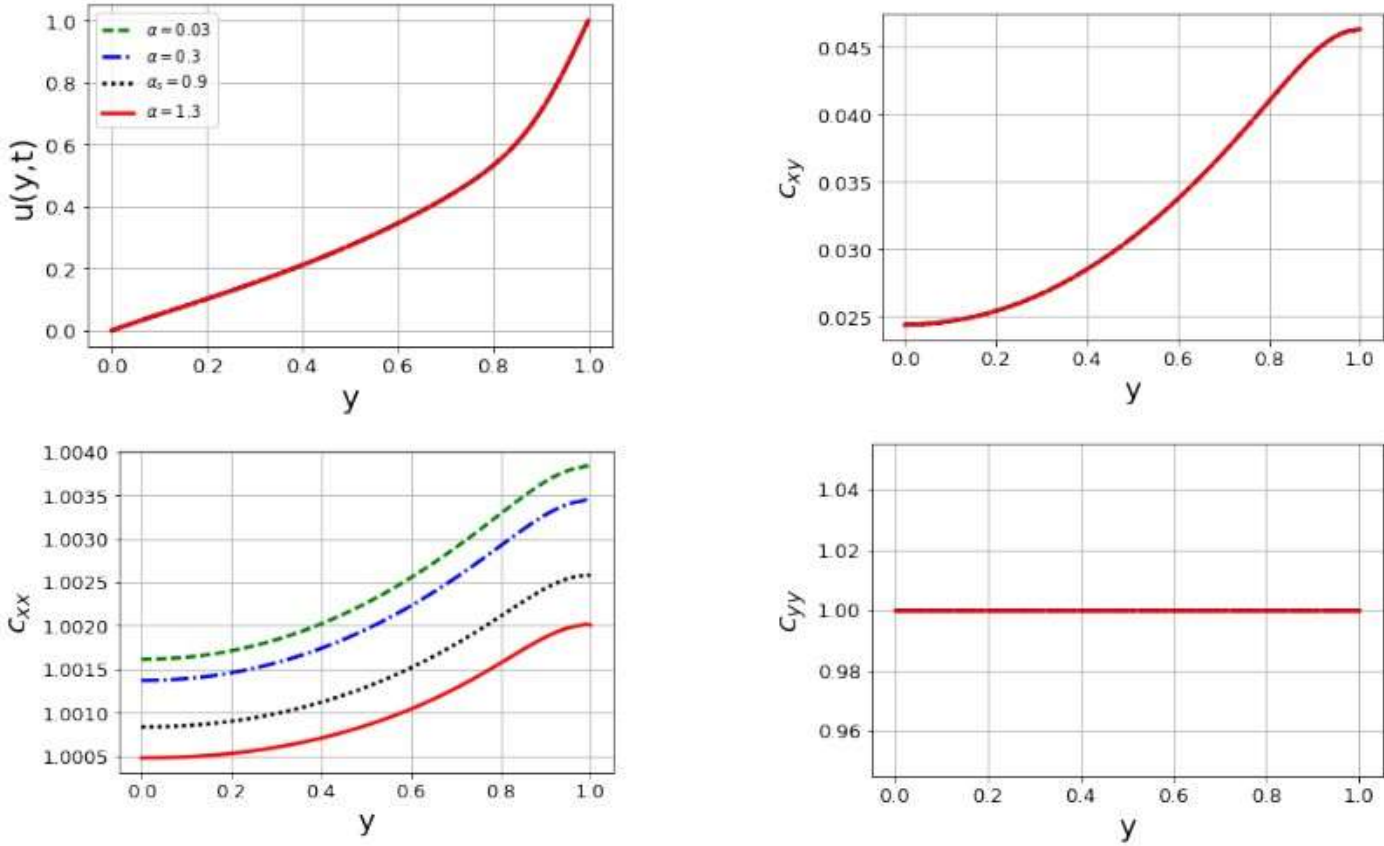

Figure 9: Variation of flow quantities with anisotropy factor $\alpha$. Parameter $\alpha$ has no observable effect on the velocity profile nor the shear stress profile, but it reduces the intensity of the normal stress $c_{x x}$ 

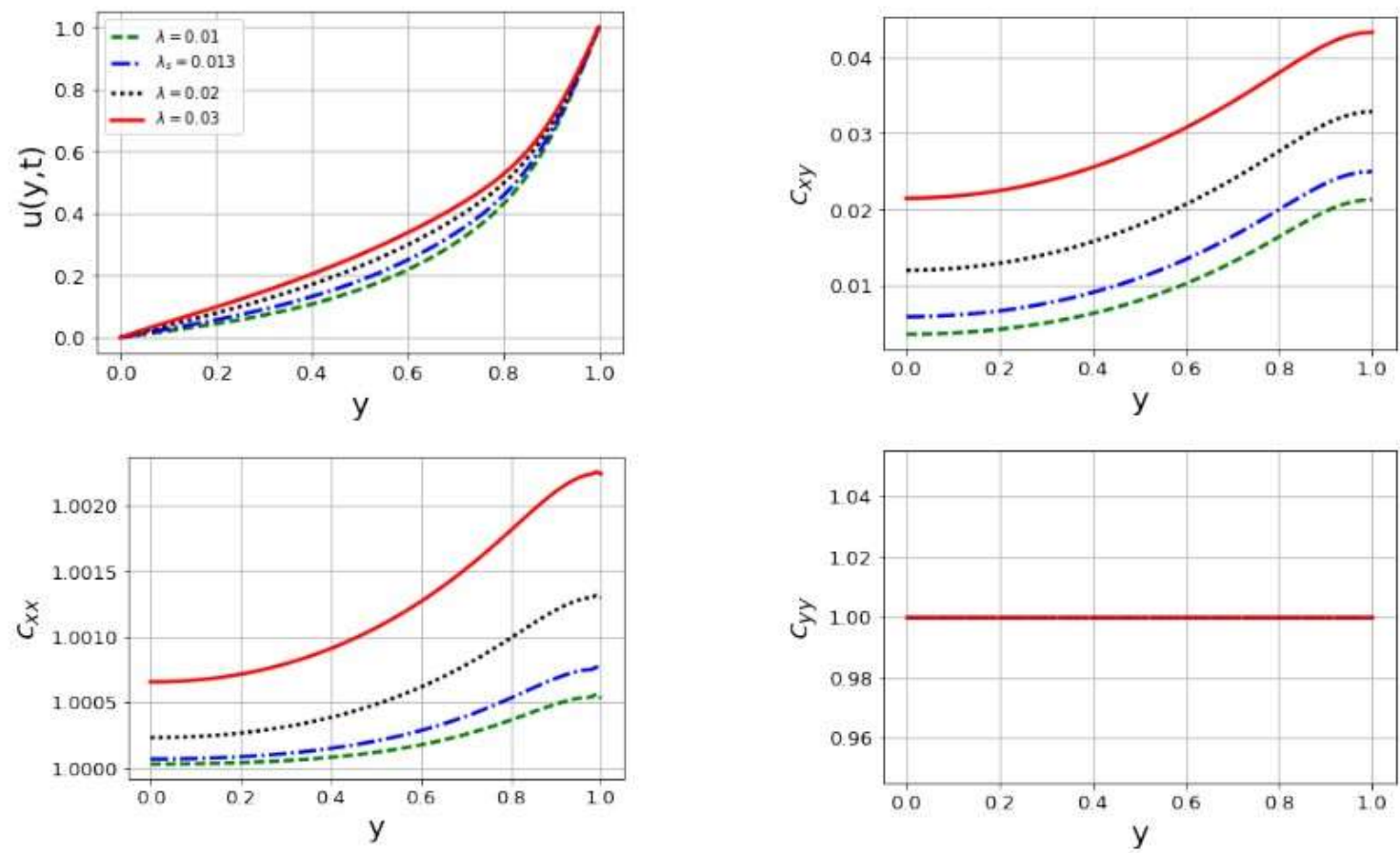

Figure 10: Variation of flow quantities with relaxation time $\lambda$. A slight increase in $\lambda$ increases both the fluid velocity and stress intensity.
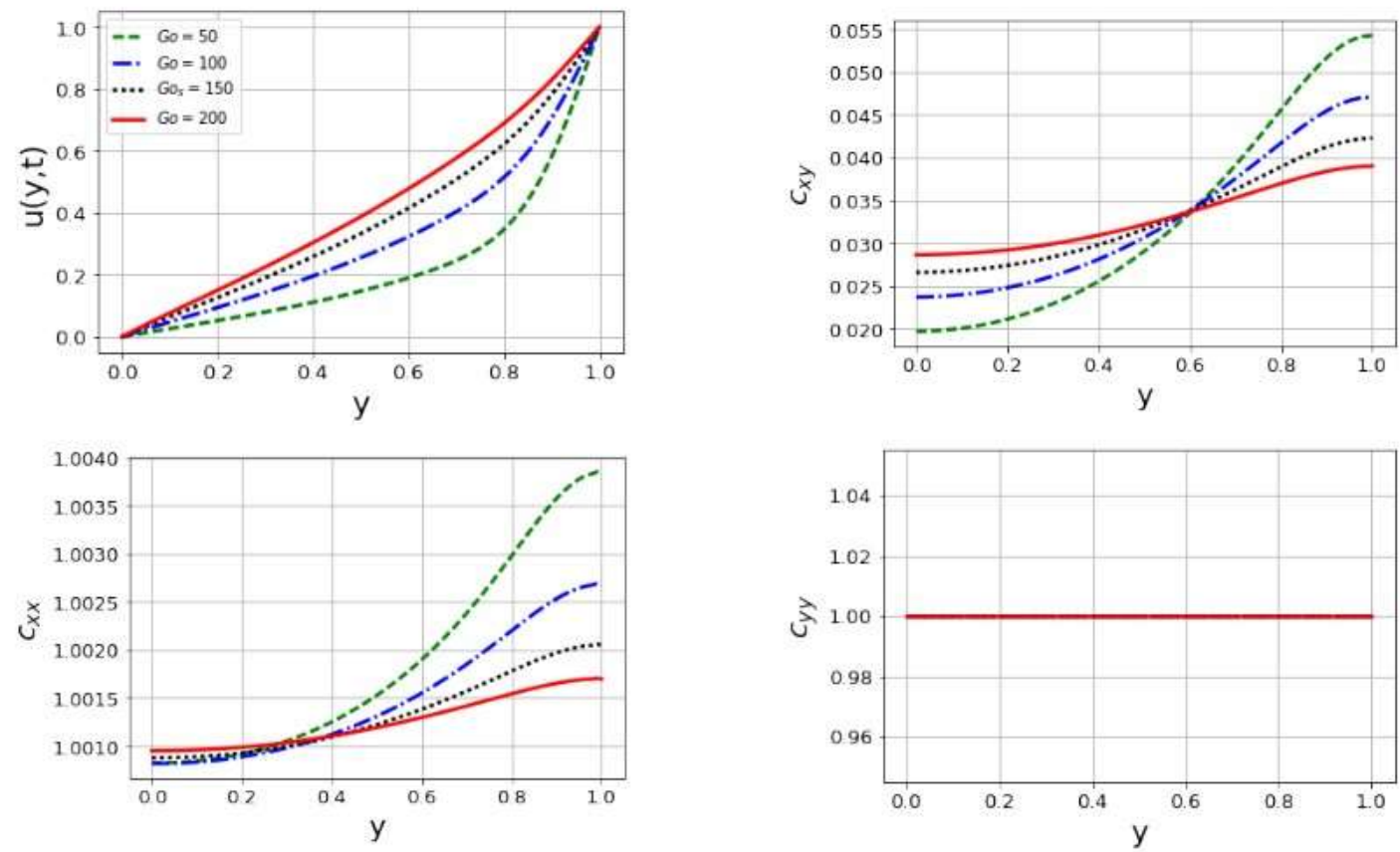

Figure 11: Variation of flow quantities with modulus of elasticity $G_{0}$. Parameter $G_{0}$ raises the velocity but lowers the intensity of stress components. 

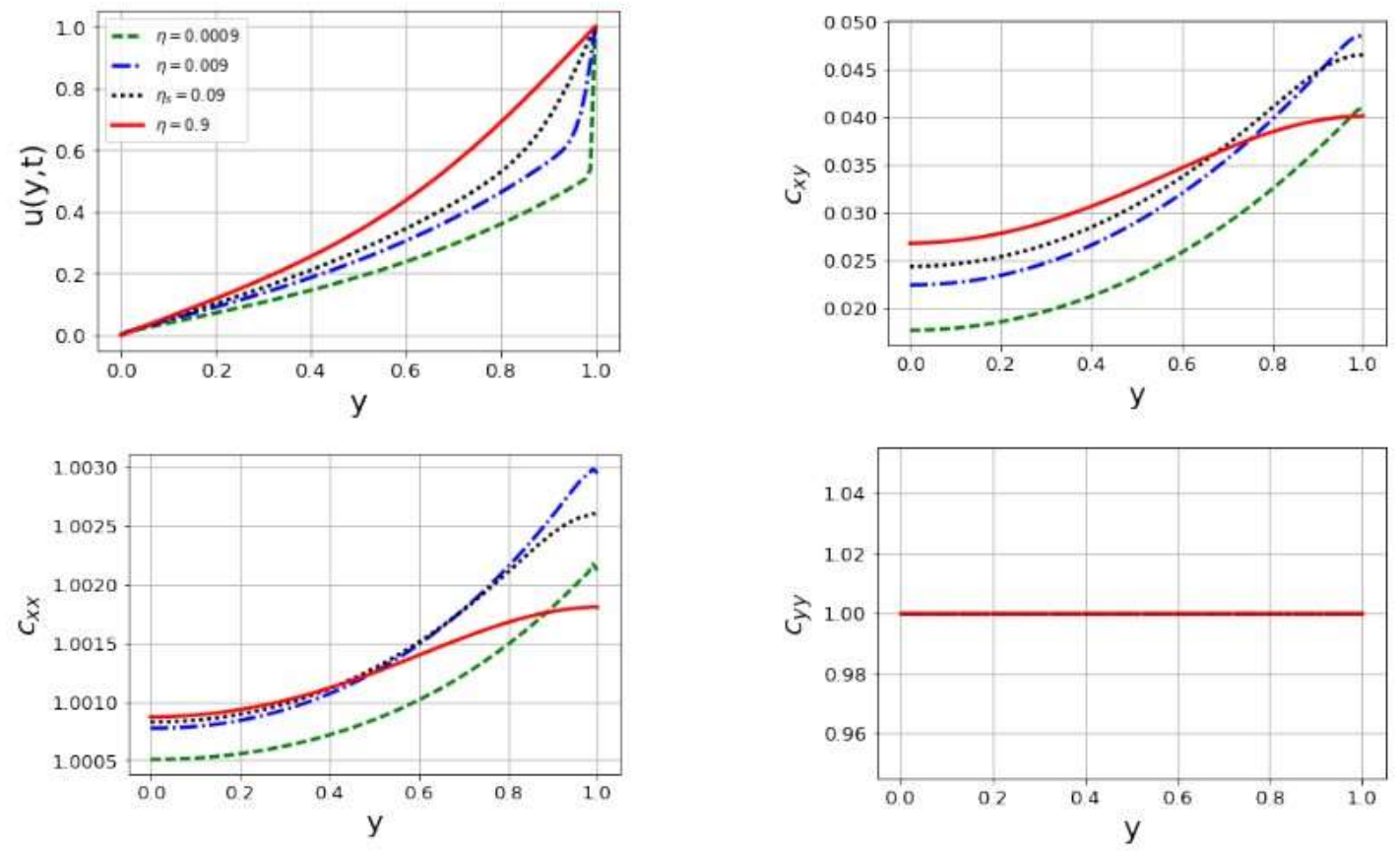

Figure 12: Variation of flow quantities with solvent viscosity $\eta_{s}$. Parameter $\eta_{s}$ raises the fluid velocity and increases the stress intensity up to $\eta=0.09 P a s$ beyond which a decrease is noticed.
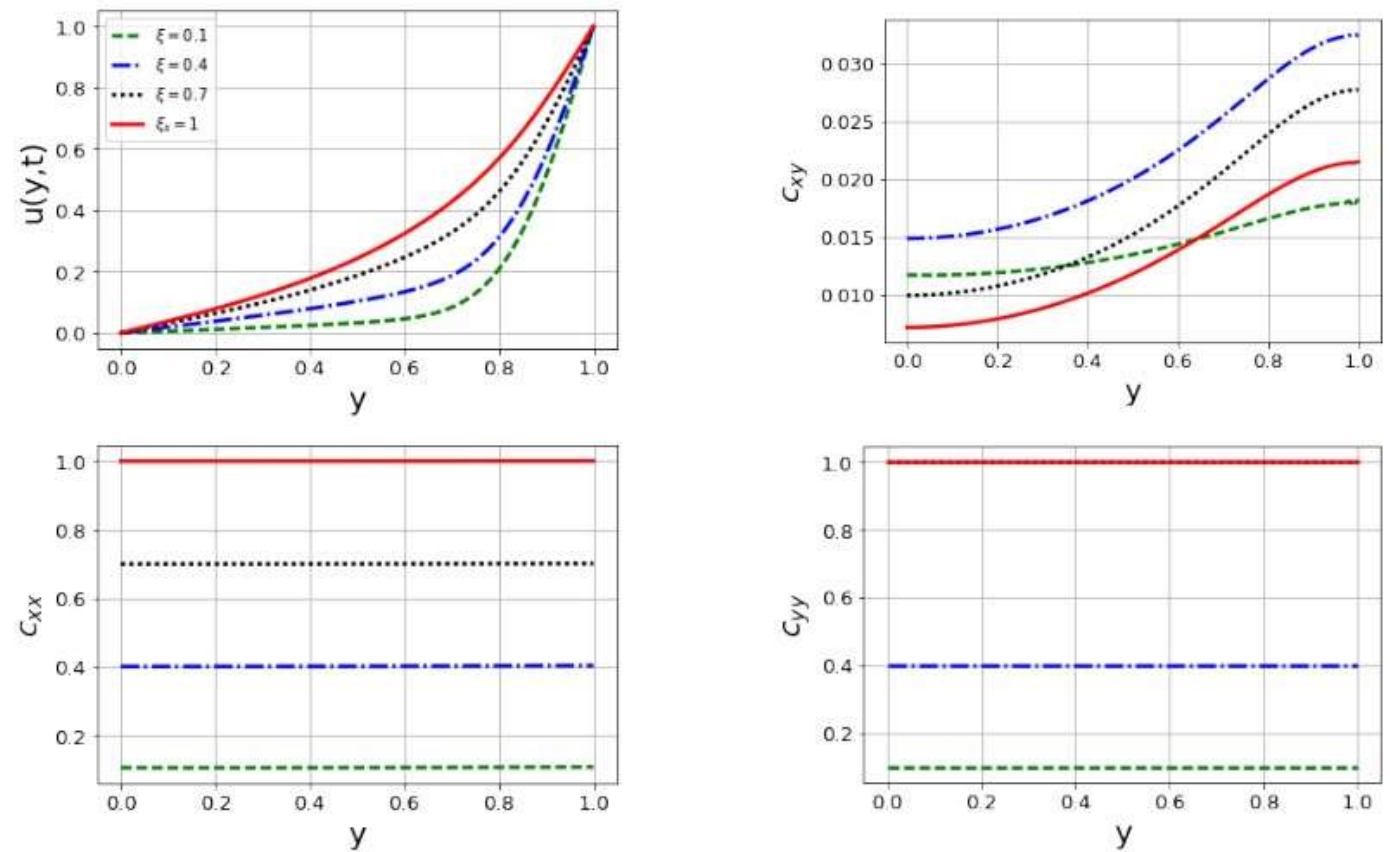

Figure 13: Variation of flow quantities with a viscoelastic parameter $\xi$. $\xi$ increases the velocity but lowers both shear and normal stresses. 

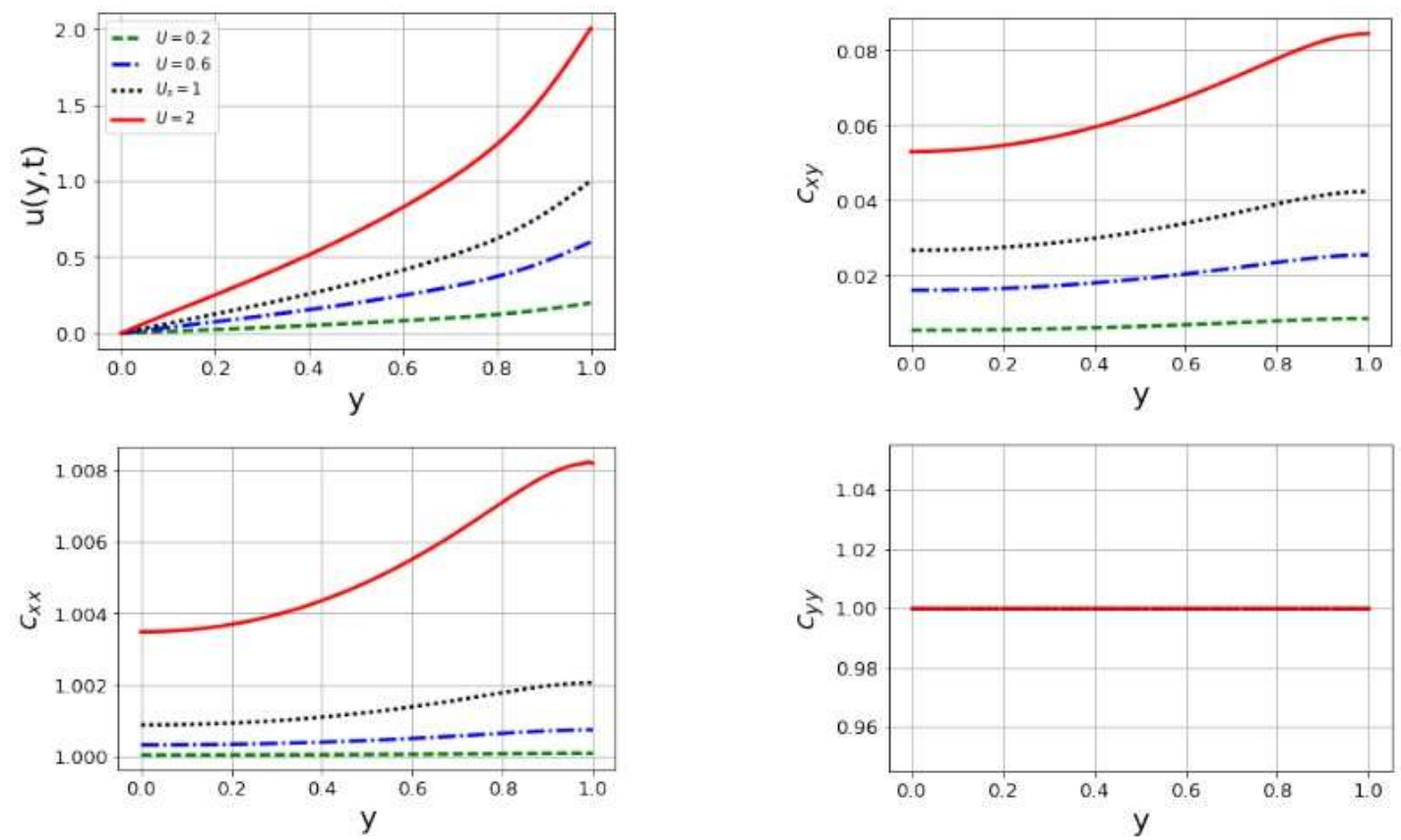

Figure 14: Variation of flow quantities with velocity $\bar{U}$ of the moving plate. Velocity increases both shear stress and normal stress. High velocity corresponds to high shear banding.

\section{CONCLUSION}

In this paper, we used the Giesekus model to investigate shear banding property of non-linear viscoelastic fluids in the plane Couette flow. Shear banding occurs in a variety of viscoelastic flows such as in shear flow of polymer melts, foams, gels, emulsions and wormlike micelles just to name a few, which exhibits non-linear rheological behavior. We observed formation of high and low shear bands when the fluid was subjected to shear forces. Low shear band corresponded to low shear rate while high-shear band corresponded to high shear rate. The Giesekus model predicted a smooth transition between linear viscoelastic and shear banding regions with a smooth edge connecting the two bands. The smooth edge corresponded to a stress plateau zone which ensures continuity of the flow profile between the two bands. For antisymmetric case two shear bands were formed while for the symmetric geometry three shear bands were formed. We observed that shear banding results to regions flowing with different velocities but with almost uniform shear stress. This explains the unstable flow behavior of viscoelastic fluids with time and space. We also observed that the concentration of visicoelastic fluid is the dominating parameter whose effect lowers the velocity but increases the intensity of shear stress. It remains to be experimentally investigated in the future whether Germann-Cook-Beris model and Maxwell model can predict similar shear banding results for a viscoelastic fluid in a Poiseuille flow.

\section{REFERENCES}

[1] Guillaume Ovarlez, St'ephane Rodts, Xavier Chateau, and Philippe Coussot. Phenomenology and physical origin of shear localization and shear banding in complex fluids. Rheologica acta, 48(8):831-844, 2009.

[2] PR Sengupta and A Kumar. A review on visco-elastic fluids: An approach to generalised model. Proceeding-Indian Natianal Science Academy Part A, 67(6):687-695, 2001.

[3] Sanjay Kumar, Iva Z Maxwell, Alexander Heisterkamp, Thomas R Polte, Tanmay P Lele, Matthew Salanga, Eric Mazur, and Donald E Ingber. Viscoelastic retraction of single living stress fibers andits impact on cell shape, cytoskeletal organization, and extracellular matrix mechanics. Biophysical journal, 90(10):3762-3773, 2006.

[4] R Byron Bird and Pierre J Carreau. A nonlinear viscoelastic model for polymer solutions and meltsi. Chemical Engineering Science, 23(5):427-434, 1968. 
[5] DV Boger. Viscoelastic flows through contractions. Annual review of fluid mechanics, 19(1):157-182,1987.

[6] Daniel D Joseph. Fluid dynamics of viscoelastic liquids, volume 84. Springer Science\& Business Media, New York, 2013.

[7] Roland Becker and Daniela Capatina. Finite element discretization of the giesekus model for polymer flows. In Numerical Mathematics and Advanced Applications 2009, pages 135-143. Springer, 2010.

[8] Brandon M Baumert and Susan J Muller. Flow visualization of the elastic taylor-couette instability in boger fluids. Rheologica Acta, 34(2):147-159, 1995.

[9] Helen Jane Wilson et al. Shear flow instabilities in viscoelastic fluids. PhD thesis, Citeseer, 1998.

[10] Ronald G Larson. Instabilities in viscoelastic flows. Rheologica Acta, 31(3):213-263, 1992.

[11]NG3 SANS Team. Shear banding in wormlike micellar solutions. Gaithersburg, 2010.

[12] MM Britton, RW Mair, RK Lambert, and PT Callaghan. Transition to shear banding in pipe and couette flow of wormlike micellar solutions. Journal of Rheology, 43(4):897-909, 1999.

[13] Patrick Le Tallec et al. Numerical methods for nonlinear viscoelastic problems in continuum mechanics. E'cole Polytechnique, Departement de M'ecanique, version, 1, 2007.

[14] Mark E Davis. Numerical methods and modeling for chemical engineers. Courier Corporation, California, 2013.

[15] MF Tom'e, GS Paulo, FT Pinho, and MA Alves. Numerical solution of the ptt constitutive equation for unsteady threedimensional free surface flows. Journal of Non-Newtonian Fluid Mechanics, 165(5-6):247-262, 2010.

[16] Charles Hirsch. Numerical computation of internal and external flows: The fundamentals of computational fluid dynamics. Elsevier, Burlington, 2007. 